\title{
Calculating current and temperature fields of HVDC grounding electrodes
}

\author{
Zheren ZHANG ${ }^{1}$, Zheng XU' ${ }^{1}$, Tao $\mathbf{X U}^{2}$
}

Abstract Current field calculation based on the resistance network method (RNM) and temperature field calculation based on the finite volume method (FVM) can be used to evaluate the performance of high-voltage direct-current (HVDC) grounding electrodes. The main idea of the two methods is to transform an electric and temperature field problems to equivalent circuit problems by dividing the 3D soil space near the grounding electrode into a suitable number of contiguous and non-overlapped cells. Each cell is represented as a central node connecting to the adjacent cells. The resistance network formed by connecting all the adjacent cells together can be solved to calculate the current field. Under the same conditions, the results calculated by the RNM are consistent with the result by CDEGS, a widely used software package for current distribution and electromagnetic field calculation. Based on the finite volume method, the temperature field results are also calculated using time domain simulation.

Keywords HVDC, Grounding electrode, Current field, Temperature field, Resistance network method, Finite volume method

CrossCheck date: 4 February 2015

Received: 30 October 2013/Accepted: 4 February 2015/

Published online: 3 June 2015

(C) The Author(s) 2015. This article is published with open access at Springerlink.com

$\triangle$ Zheng XU

xuzheng007@zju.edu.cn

Zheren ZHANG

zheren.zhang@hotmail.com

1 Department of Electrical Engineering, Zhejiang University, Hangzhou 310027, China

2 Zhejiang Electric Power Corporation Research Institute, Hangzhou 310014, China

\section{Introduction}

HVDC transmission is a common technology for longdistance and high-capacity power transmission $[1,2]$. As an important part of a HVDC system, the grounding electrode provides the system with a path for DC current or unbalanced current $[3,4]$. A variety of factors should be considered in designing the DC grounding electrode, such as environment, cost and technology [5].

The design of the HVDC grounding electrode mainly concerns the current field and temperature field $[6,7]$. The current field determines the shape and the size of the grounding electrode to meet the safety standards such as step voltage and serves as prior knowledge for calculating the temperature field [8]. Due to environmental and safety considerations, the engineers are also interested in the temperature field in the ground surrounding the electrode

Detailed research has been reported in the literature on calculating current and temperature fields for HVDC grounding electrodes. Generally speaking, the complex image method has a high accuracy and is simple in operation [9]. However, this method requires vertically stratified soil and is not applicable when the soil resistivity varies in all directions. The boundary element method for calculating the current field is relatively complicated in its formulation and calculation, and the finite element method requires large memory [10]. These drawbacks also exist for the finite difference method for calculating the temperature field.

Considering the limitations of the present methods for calculating current and temperature fields, this paper describes a new resistance network method (RNM) for calculating the current field and a finite volume method (FVM) for calculating the temperature field, which are both relatively simple in formulation. 


\section{Current field and temperature field calculating method}

\subsection{Basic theory}

Neither the RNM nor the FVM involve complex analysis. The basic concept of the two methods is to divide the 3D soil space near the grounding electrode into non-overlapping cells. Then, each cell is considered as a node, and connecting all the nodes together forms a network. After defining the properties of nodes and connections, the current field and the temperature field can be calculated simply by solving the network problem.

The RNM and the FVM allow the soil resistivity to vary in all directions because of the arbitrary subdivision of the soil space. As a result, they can be applied to different types of grounding structure.

Generally speaking, the steps of the RNM and the FVM include: 1) defining and subdividing the problem domain and 2) forming and solving the network. These steps are explained in detail in the following sections based on the most commonly used ring shape and track shape for grounding electrodes.

\subsection{Problem domain definition and subdivision}

The current injected into the grounding electrode will flow to infinity, and the current field analysis in theory is an infinite boundary problem. However, in practical situations, when the grounding current flows a large enough distance, the soil potential and the electric field will effectively reduce to zero. For all finite element methods, the problem domain for current field calculation must be defined with a finite boundary in order to make the analysis practicable. So, the current field calculation is limited within the defined finite boundary, and the soil potential is regarded as zero outside the boundary. This simplification is also adopted in the calculation of temperature field, and the temperature is regarded as constant outside the boundary.

The definition and subdivision of the problem domain has a direct influence on the speed and accuracy of the field calculation. Normally, the defined domain corresponds to the shape of the grounding electrode, so as to be subdivided easily. For many useful electrode geometries the problem domain can be a right cylinder or a right prism containing the grounding electrode. The top surface of the problem domain represents the surface of the soil, and the central axis of it coincides with that of the electrode, while the cross section shape matches the electrode shape. For a ringshaped grounding electrode, the cross section shape is a circle; for a track-shaped grounding electrode, the cross section shape is a track, consisting of two straight parts and two semicircular parts. In an analogous way, for other types of grounding electrode, the cross section shape can be to match the shape of grounding electrode. Fig. 1 and Fig. 2 show the finite problem domains of a ring-shaped and a track-shaped grounding electrode.

According to experiences gained from many practical projects, it is reasonable to set the radius $r_{d}$ for the cross section as $100 \mathrm{~km}$ and the height $h_{d}$ of the problem domain as $100 \mathrm{~km}$ when calculating the current field. The curvature of the Earth can be ignored at this scale. In contrast, the radius $r_{d}$ and height $h_{d}$ are both set to $1 \mathrm{~km}$ when calculating the temperature field, since the temperature variation soon becomes small compared to the background variability of temperature, whereas electric fields are detectable for very long distances.

Fan-shaped cells and rectangular prism cells are two basic subdivisions of these problem domains. The fanshaped cell is applied to the region with circular arc boundaries. Correspondingly, a cylindrical coordinate system is adopted. The origin of the coordinate system is the center of the circular arc. The $Z$ axis of the coordinate system is in the vertical direction, and the $r, \theta$ surface of the coordinate system is parallel to the surface of the soil.

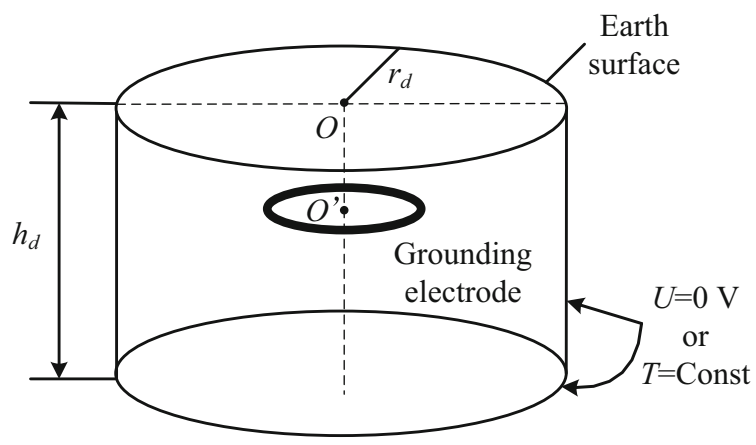

Fig. 1 The problem domain for a ring-shaped grounding electrode

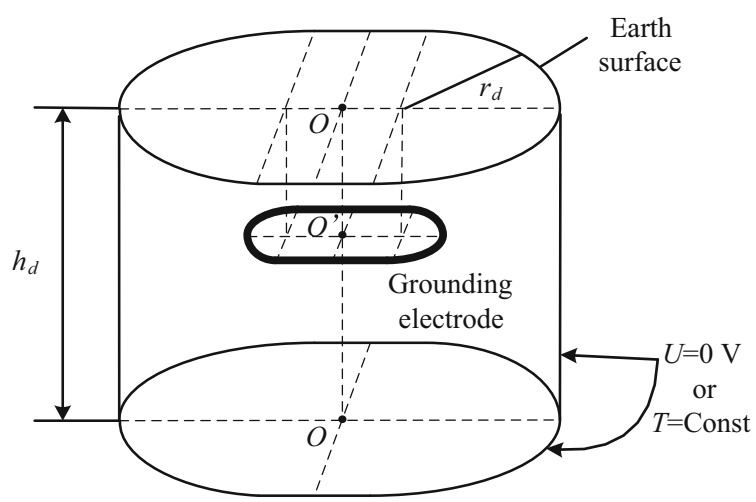

Fig. 2 The problem domain for a track-shaped grounding electrode 
According to the cylindrical coordinate system, a circular cylindrical domain or a semi-circular cylindrical subdomain is divided into fan-shaped cells in the depth direction $Z$, the radius direction $r$ and the angular direction $\theta$, as shown in Fig. 3. Suppose this cell is represented by its central node $P$, then nodes $1-6$ are the central nodes of the adjacent cells at each face. The difference (absolute value) between $P$ and $1(2)$ is $\delta r_{1}\left(\delta r_{2}\right)$, the difference between $P$ and 3 (4) is $\delta \theta_{3}\left(\delta \theta_{4}\right)$, and the difference between $P$ and 5 (6) is $\delta z_{5}\left(\delta z_{6}\right)$. The volume of this cell is $V$, and the other symbols are shown in Fig. 3.

The rectangular cell is applied to the region with straight boundaries. Correspondingly, a 3D Cartesian coordinate system is adopted. The origin of the coordinate system is the center of the area within straight boundaries. The $Z$ direction of the coordinate system is the vertical direction, and the $X, Y$ surface of the coordinate system is the surface of the soil. The rectangular subdomain is then divided into rectangular cells in directions $X, Y$ and $Z$, as is shown in Fig. 4. Suppose this cell is represented by its central node $P$, then nodes $1-6$ are the central nodes of the

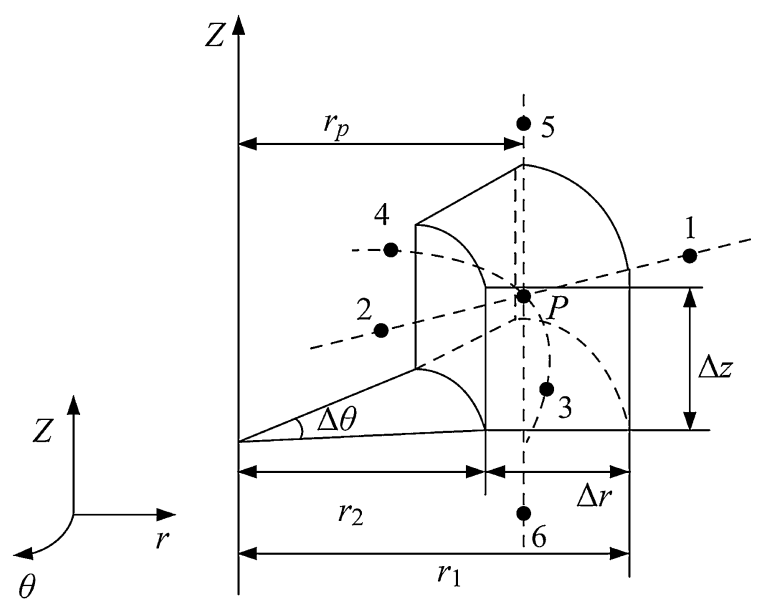

Fig. 3 Schematic diagram of a fan-shaped cell

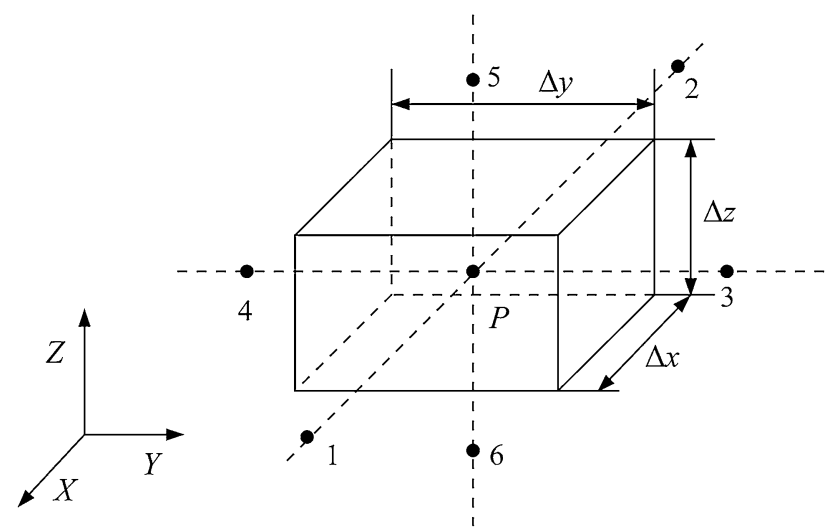

Fig. 4 Schematic diagram of a rectangular prism cell adjacent cells at each face. The difference (absolute value) between $P$ and 1 (2) is $\delta x_{1}\left(\delta x_{2}\right)$, the difference between $P$ and $3(4)$ is $\delta y_{3}\left(\delta y_{4}\right)$, and the difference between $P$ and 5 (6) is $\delta z_{5}\left(\delta z_{6}\right)$. The volume of this cell is $V$, and the other symbols are shown in Fig. 4.

According to the analysis above, for many useful electrode geometries, the problem domain can be subdivided using fan-shaped cells and rectangular prism cells. For a ring shaped grounding electrode, only fan-shaped cells are needed. For the track-shaped grounding electrode, both fan-shaped and rectangular prism cells are needed.

\subsection{Network formation and matrix representation to obtain the current field}

The equivalent resistance of the cells in each coordinate direction is derived by integrating the formula for conductor resistance:

$R=\rho \frac{l}{S}$

where $\rho$ is the resistivity of the conductor; $l$ is the length of the conductor; $S$ is the cross-sectional area of the conductor. The resistivity is assumed to be constant within each cell.

According to Fig. 3, the equivalent resistances for the fan-shaped cell in the radius direction $r$, the angular direction $\theta$ and the depth direction $Z$ are derived as follows:

$R_{r}=\rho_{F} \int_{r_{2}}^{r_{1}} \frac{\mathrm{d} r}{\Delta z \Delta \theta r}=\frac{\rho_{F}}{\Delta z \Delta \theta} \ln \frac{r_{1}}{r_{2}}$

$R_{\theta}=\rho_{F} \frac{1}{\int_{r_{2}} \frac{\Delta \mathrm{zd} r}{r \Delta \theta}}=\frac{\rho_{F} \Delta \theta}{\Delta z \ln \frac{r_{1}}{r_{2}}}$

$R_{Z}=\rho_{F} \frac{\Delta z}{\int_{r_{2}} r \Delta \theta \mathrm{d} r}=\frac{2 \rho_{F} \Delta z}{\Delta \theta\left(r_{1}^{2}-r_{2}^{2}\right)}$

where $\rho_{F}$ is the soil resistivity of the fan-shaped cell; $r_{1}$ and $r_{2}$ are the outer radius and the inner radius of the cell; $\Delta \theta$ and $\Delta z$ are the fan angle and the thickness of the cell.

According to Fig. 4, the equivalent resistances for the rectangular cell in the direction $X, Y$ and $Z$ are derived as follows:

$$
\begin{aligned}
& R_{X}=\frac{\rho_{R} \Delta x}{\Delta y \Delta z} \\
& R_{Y}=\frac{\rho_{R} \Delta y}{\Delta x \Delta z} \\
& R_{Z}=\frac{\rho_{R} \Delta z}{\Delta x \Delta y}
\end{aligned}
$$


where $\rho_{R}$ is the soil resistivity of the rectangular cell; $\Delta x$ is the length of the cell; $\Delta y$ is the width of the cell; $\Delta z$ is the thickness of the cell.

Figure 5 shows the equivalent resistances within a rectangular cell. To form the equivalent resistance network of a group of cells, a rectangular cell is represented by node $P$ at its center, and connects to the adjacent cells by half its equivalent resistance in the direction towards each connecting face.

By connecting all adjacent cells in the problem domain, the equivalent resistance network can be formed. Figure 6 illustrates the equivalent resistance network of some adjacent rectangular prism cells ( $X Y$ plane view). A fan-shaped cell is also represented by node $P$ at its center, and is incorporated in the network in the same way, using half its equivalent resistance in each coordinate direction. Thus the full problem domain can be converted to an equivalent resistance network.

To solve for the potential field it is convenient to use the admittance matrix $\boldsymbol{Y}_{R}$ of the resistance network. The network can be described as follows:

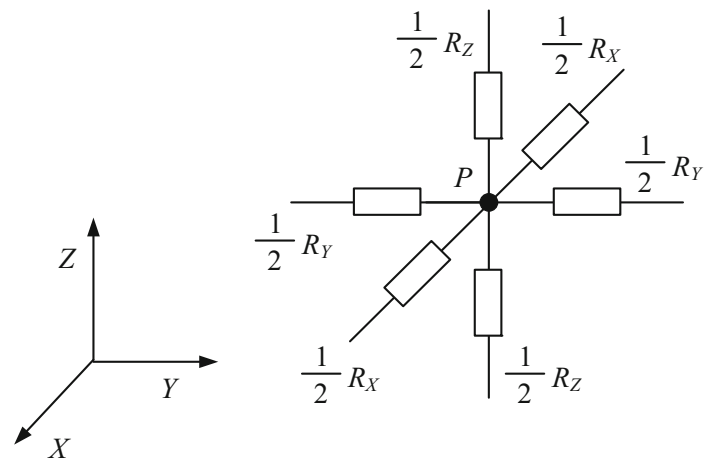

Fig. 5 The equivalent resistances of a rectangular prism cell

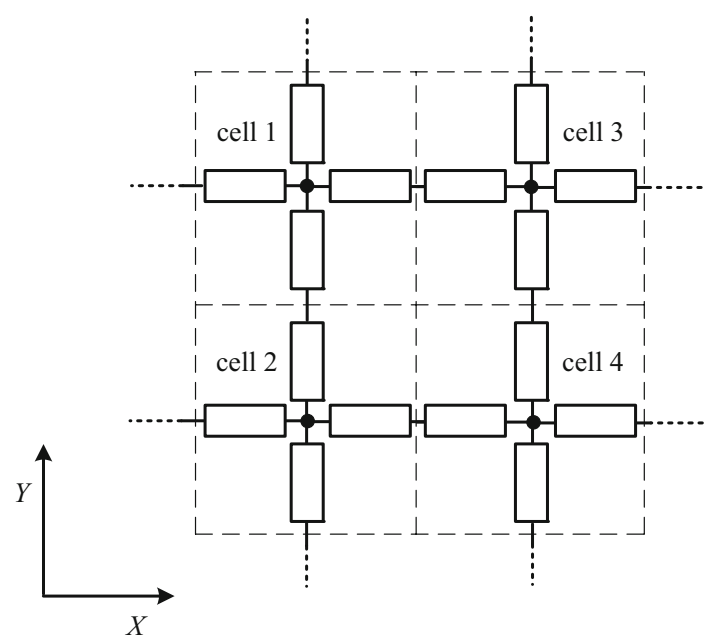

Fig. 6 The equivalent resistance network of some adjacent cells
$\boldsymbol{Y}_{R} \boldsymbol{U}_{R}=\boldsymbol{I}_{R}$

where $\boldsymbol{U}_{R}$ is the voltage vector of all nodes in the resistance network and $\boldsymbol{I}_{R}$ is the injection current vector of the network.

In order to simplify the analysis, the grounding electrode is regarded as an equipotential system, which means that all the nodes belonging to the grounding electrode itself have the same voltage. In addition, the injection currents are from the electrode nodes, and not from the soil nodes.

Therefore, (8) can be reordered to separate the electrode nodes and the soil nodes, giving block matrices as follows:

$\left[\begin{array}{ll}\boldsymbol{Y}_{11} & \boldsymbol{Y}_{12} \\ \boldsymbol{Y}_{21} & \boldsymbol{Y}_{22}\end{array}\right]\left[\begin{array}{l}\boldsymbol{U}_{1} \\ \boldsymbol{U}_{2}\end{array}\right]=\left[\begin{array}{c}\boldsymbol{I}_{M} \\ \mathbf{0}\end{array}\right]$

where $\boldsymbol{Y}_{11}$ and $\boldsymbol{Y}_{22}$ are the self-admittance matrices of the electrode nodes and the soil nodes; $\boldsymbol{Y}_{12}$ and $\boldsymbol{Y}_{21}$ are the mutual-admittance matrices between the electrode nodes and the soil nodes; $\boldsymbol{U}_{1}$ and $\boldsymbol{U}_{2}$ are the voltage vectors of the electrode nodes and the soil nodes; and $\boldsymbol{I}_{M}$ is the injection current vector of the electrode nodes. The injection current vector of the soil nodes is $\mathbf{0}$.

\subsection{Network formation and matrix representation to obatin the temperature field}

The governing equation that describes the temperature field is as follows:

$\nabla^{2} T+\frac{\rho}{\lambda} J^{2}=\frac{C}{\lambda} \frac{\partial T}{\partial t}$

where $T$ and $J$ are the temperature field and the current density in the problem domain; $\rho, C$ and $\lambda$ are respectively the resistivity, the thermal capacity and the thermal conductivity of the soil; $t$ is time.

With the help of the FVM [11], (10) becomes the following form:

$a_{P} T_{P}=a_{1} T_{1}+a_{2} T_{2}+a_{3} T_{3}+a_{4} T_{4}+a_{5} T_{5}+a_{6} T_{6}+b$

where

$$
\left\{\begin{array}{l}
a_{1}=\frac{\Delta y \Delta z}{\delta x_{1} / \lambda}, a_{2}=\frac{\Delta y \Delta z}{\delta x_{2} / \lambda}, a_{3}=\frac{\Delta x \Delta z}{\delta y_{3} / \lambda} \\
a_{4}=\frac{\Delta x \Delta z}{\delta y_{4} / \lambda}, a_{5}=\frac{\Delta x \Delta y}{\delta z_{5} / \lambda}, a_{6}=\frac{\Delta x \Delta y}{\delta z_{6} / \lambda} \\
a_{P}=a_{1}+a_{2}+a_{3}+a_{4}+a_{5}+a_{6}+C \Delta x \Delta y \Delta z /(\Delta t \lambda) \\
b=J_{P}^{2} \rho_{P} \Delta x \Delta y \Delta z+C \Delta x \Delta y \Delta z /(\Delta t \lambda) T_{P}^{(n-1)}
\end{array}\right.
$$

for the rectangular prism cells, or: 


$$
\left\{\begin{array}{l}
a_{1}=\frac{r_{1} \Delta \theta \Delta z}{\delta r_{1} / \lambda}, a_{2}=\frac{r_{2} \Delta \theta \Delta z}{\delta r_{2} / \lambda}, a_{3}=\frac{\Delta r \Delta z}{r_{P} \delta \theta_{3} / \lambda} \\
a_{4}=\frac{\Delta r \Delta z}{r_{P} \delta \theta_{4} / \lambda}, a_{5}=\frac{r_{P} \Delta r \Delta \theta}{\delta z_{5} / \lambda}, a_{6}=\frac{r_{P} \Delta r \Delta \theta}{\delta z_{6} / \lambda} \\
a_{P}=a_{1}+a_{2}+a_{3}+a_{4}+a_{5}+a_{6}+C \frac{r_{P} \Delta r \Delta \theta \Delta z}{\Delta t \lambda} \\
b=J_{P}^{2} \rho_{P} r_{P} \Delta r \Delta \theta \Delta z+C \frac{r_{P} \Delta r \Delta \theta \Delta z}{\Delta t \lambda} T_{P(\text { prev })}
\end{array}\right.
$$

for the fan-shaped cells.

Here in (12)-(13), $T_{P(\text { prev })}$ is the temperature of node $P$ at the previous time point, and $T_{1}, T_{2}, \ldots, T_{6}$ and $T_{P}$ are the updated temperatures of nodes $1-6$ and $P$.

For the boundary cells of the problem domain, some minor changes will be made in (11). For the cells that have convective heat transfer, the equation describing the central node and the adjacent nodes becomes:

$a_{P}^{\prime} T_{P}=a_{1} T_{1}+a_{2} T_{2}+a_{3} T_{3}+a_{4} T_{4}+a_{6} T_{6}+b^{\prime}$

For the rectangular cells where convective heat transfer exists on the surface between nodes $P$ and 5 as shown in Fig. 3, some variables are defined as:

$$
\left\{\begin{array}{l}
a_{P}^{\prime}=a_{1}+a_{2}+a_{3}+a_{4}+a_{6}+A /\left(1 / h+\delta x_{w} / \lambda\right) \\
b^{\prime}=J_{P}^{2} \rho_{P} \Delta x \Delta y \Delta z+C \frac{\Delta x \Delta y \Delta z}{\Delta t \lambda} T_{P(\text { prev })}+\frac{A T_{\text {air }}}{1 / h+\delta x_{w} / \lambda} \\
A=\Delta x \Delta y
\end{array}\right.
$$

where $A$ is the area of the convective surface; $\delta x_{W}$ is the distance between node $P$ and the convective surface; $h$ is the convective coefficient. The results are similar to the fan-shaped cells.

\subsection{Solving the network equations}

When (9) is expanded, the following equations are obtained:

$$
\begin{aligned}
& \boldsymbol{Y}_{11} \boldsymbol{U}_{1}+\boldsymbol{Y}_{12} \boldsymbol{U}_{2}=\boldsymbol{I}_{M} \\
& \boldsymbol{Y}_{21} \boldsymbol{U}_{1}+\boldsymbol{Y}_{22} \boldsymbol{U}_{2}=\mathbf{0}
\end{aligned}
$$

Since the admittance matrix of the network is known, $\boldsymbol{Y}_{21}$ and $\boldsymbol{Y}_{22}$ in (17) are known. However, the electrode nodes' voltage vector $\boldsymbol{U}_{1}$ and the soil nodes' voltage vector $\boldsymbol{U}_{2}$ are unknown. Noting the linearity of (9) and the equalpotentiality of electrode nodes, it is reasonable to preset $\boldsymbol{U}_{1}$ to the assumed or known voltage of the electrode.

With a preset electrode voltage value (such as $1000 \mathrm{~V}$ ) for voltage vector $\boldsymbol{U}_{1}$ in (17), the voltage vector of the soil nodes $\boldsymbol{U}_{2}$ can be calculated. The injection current vector $\boldsymbol{I}_{M}$ corresponding to the preset voltage is acquired by substituting $\boldsymbol{U}_{1}$ and $\boldsymbol{U}_{2}$ into (16). The injection current of the system equals the elements sum of vector $\boldsymbol{I}_{M}$ :

$I_{C}=\sum_{i=1}^{n} I_{i}$

where $n$ is the total number of the electrode nodes; $I_{i}$ is the $i^{\text {th }}$ element of vector $\boldsymbol{I}_{M}$. Assuming the real grounding current is $I_{G}$, the linear correction factor is the ratio of the real grounding current value and the calculated injection current value, $k_{L}=I_{G} / I_{C}$. By multiplying all the calculated node voltages by $k_{L}$, the real voltage value of all the nodes in the network is obtained, consistent with the known injection current.

To calculate the temperature field, (11)-(15) are represented as a matrix equation:

$\boldsymbol{A T}=\boldsymbol{B}$

The node temperatures $\boldsymbol{T}$ can be calculated by solving (19).

This completes the description of current field and temperature field calculation using the RNM and the FVM. The following section verifies these methods by comparison of numerical results.

\section{Case study}

The case studied is a HVDC project with $\pm 800 \mathrm{kV}$ nominal DC voltage. Under the same conditions, the current field results [12, 13] calculated by the RNM are compared with the results of the CDEGS software package [14] in order to verify the validity of the method.

As shown in Fig. 7, the grounding electrode comprises two conductors, which are both track-shaped.

Cross Section 1 represents the middle of the straight part, cross Section 2 represents the intersection of the straight and curved parts, and cross Section 3 represents the middle of the curved part. Table 1 shows the relevant

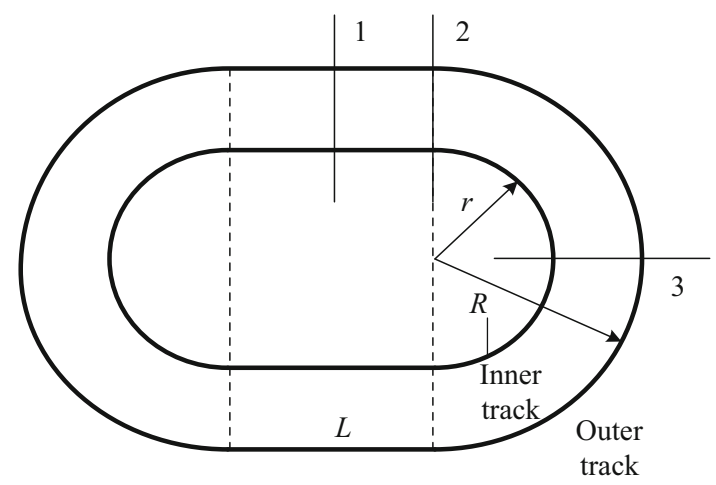

Fig. 7 Schematic diagram of a double-track shaped electrode 
Table 1 Relevant parameters of the test system

\begin{tabular}{ll}
\hline Straight length $(\mathrm{m})$ & 800 \\
\hline Curve radius $(\mathrm{m})$ & Inner: $175 /$ Outer: 250 \\
Electrode depth $(\mathrm{m})$ & Inner: 3.0/Outer: 3.5 \\
Coke side length (m) & Inner: 0.8/Outer: 0.9 \\
Rated current $I_{G N}(\mathrm{~A})$ & 4700 \\
Soil thermal capacity $C\left(\mathrm{~J} /\left(\mathrm{m}^{3} \cdot{ }^{\circ} \mathrm{C}\right)\right)$ & $1.0 \times 10^{6}$ \\
Soil thermal conductivity $\lambda\left(\mathrm{W} /\left(\mathrm{m} \cdot{ }^{\circ} \mathrm{C}\right)\right)$ & 0.42 \\
Convective coefficient $h\left(\mathrm{~W} /\left(\mathrm{m}^{2} \cdot{ }^{\circ} \mathrm{C}\right)\right)$ & 1.0 \\
Ambient air temperature $\left({ }^{\circ} \mathrm{C}\right)$ & 20 \\
Ambient soil temperature $\left({ }^{\circ} \mathrm{C}\right)$ & 25 \\
Period for mono-polar operation (day) & 120 \\
\hline
\end{tabular}

Table 2 Soil resistivity data of the test system

\begin{tabular}{lllccl}
\hline No. & $\begin{array}{l}\text { Depth } \\
(\mathrm{m})\end{array}$ & $\begin{array}{l}\text { Resistivity } \\
(\Omega \cdot \mathrm{m})\end{array}$ & No. & $\begin{array}{l}\text { Depth } \\
(\mathrm{m})\end{array}$ & $\begin{array}{l}\text { Resistivity } \\
(\Omega \cdot \mathrm{m})\end{array}$ \\
\hline 1 & 7 & 204 & 6 & 32 & 231 \\
2 & 8 & 139 & 7 & 37 & 278 \\
3 & 18 & 103 & 8 & 46 & 292 \\
4 & 26 & 130 & 9 & 73 & 310 \\
5 & 30 & 150 & 10 & $>73$ & 342 \\
\hline
\end{tabular}

parameters of the system. Table 2 shows the soil resistivity data for the region of the electrode. To provide enough distance for the potential field and the temperature field to become constant, as assumed in Section 2.3, the problem domain for the RNM and the FVM has curve radius $X$, straight length $Y$, and depth $Z$, and this is divided into a total of about 1 million fan-shaped and rectangular prism cells.

Figure 8 shows the current field results for the grounding electrode. The deviation between the results calculated by the RNM and CDEGS is very small (within 5\%), which shows the validity of the RNM. Besides, the maximal step voltage and the surface current density are both within the safety limit [15], which meets the requirements of the project.

Figure 9 shows the temperature field results calculated using the FVM at a depth of $3.5 \mathrm{~m}$ after 120 days of monopolar operation. Figure 10 shows the temperature variation with time at the hottest point (as marked in Fig. 9) under different soil thermal conductivities. Because the CDEGS software does not calculate the temperature field, the FVM results are compared with results based on previously published formulas [16].

Comparing the FVM time-domain simulation results and the calculated results by published formulas in Fig. 10, it can be seen that the time-domain temperatures are much smaller, because the published formulas are based on the assumption that there is no heat conduction in the problem
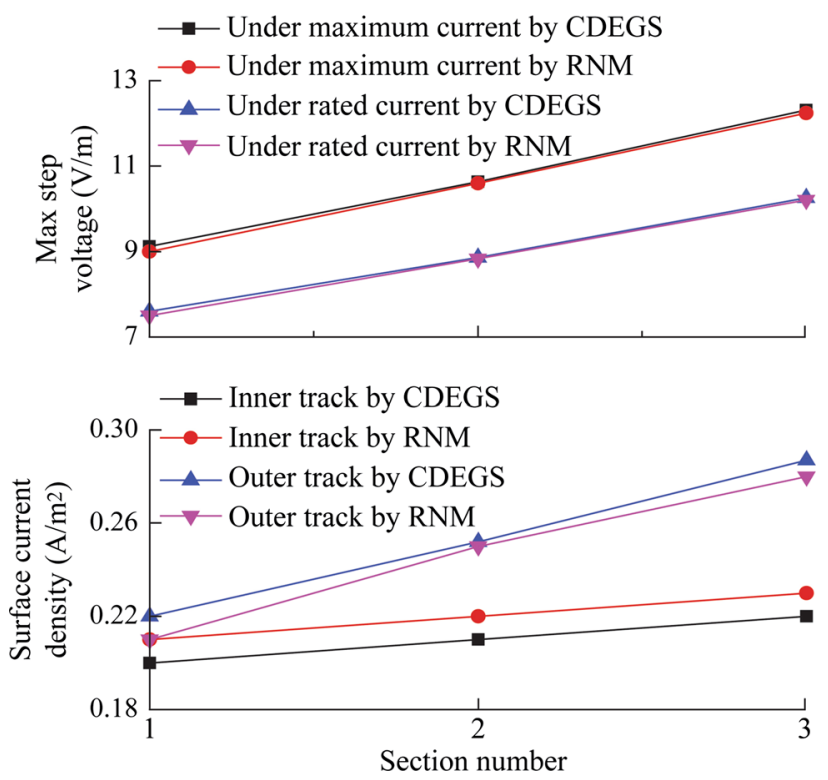

Fig. 8 Current field results of the grounding electrode

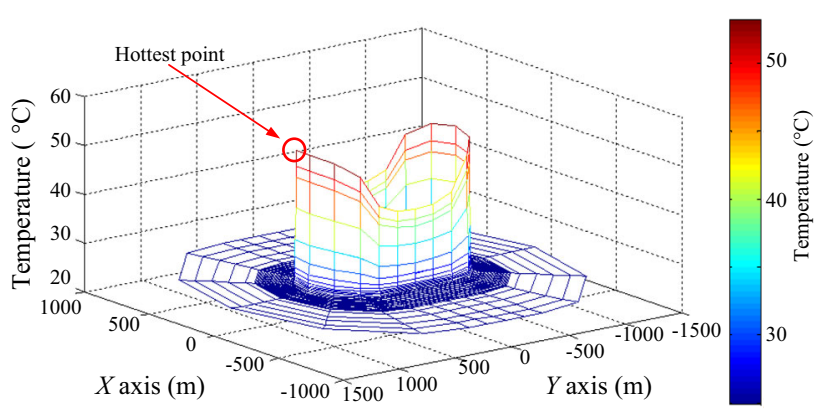

Fig. 9 Temperature field results by the FVM at a depth of $3.5 \mathrm{~m}$

domain. However, the heat conduction is taken into account in the time domain simulation. It can be concluded from Fig. 10 that soil thermal conductivity must be taken into account, even for quite low thermal conductivities (e.g. $\lambda=0.1 \mathrm{~W} /\left(\mathrm{m} \cdot{ }^{\circ} \mathrm{C}\right)$ ), to avoid unrealistic amounts of heat storage in the surrounding soil. A full study should also consider when electrode-induced heat flows become insignificant compared to the underlying soil heat flows.

For practical HVDC projects, the soil temperature should always be kept below the boiling point of water, which puts a demand on the current density in the soil (the dimension of the grounding electrode). As mentioned above, the highest temperature calculated by the previously published formulas is always higher than the time-domain simulation result. In this regard, the time-domain simulation based on FVM provides an important alternative way to check the temperature requirements at the basic design stage of HVDC projects, which could lead to better use of the grounding electrode without extending its dimension. 

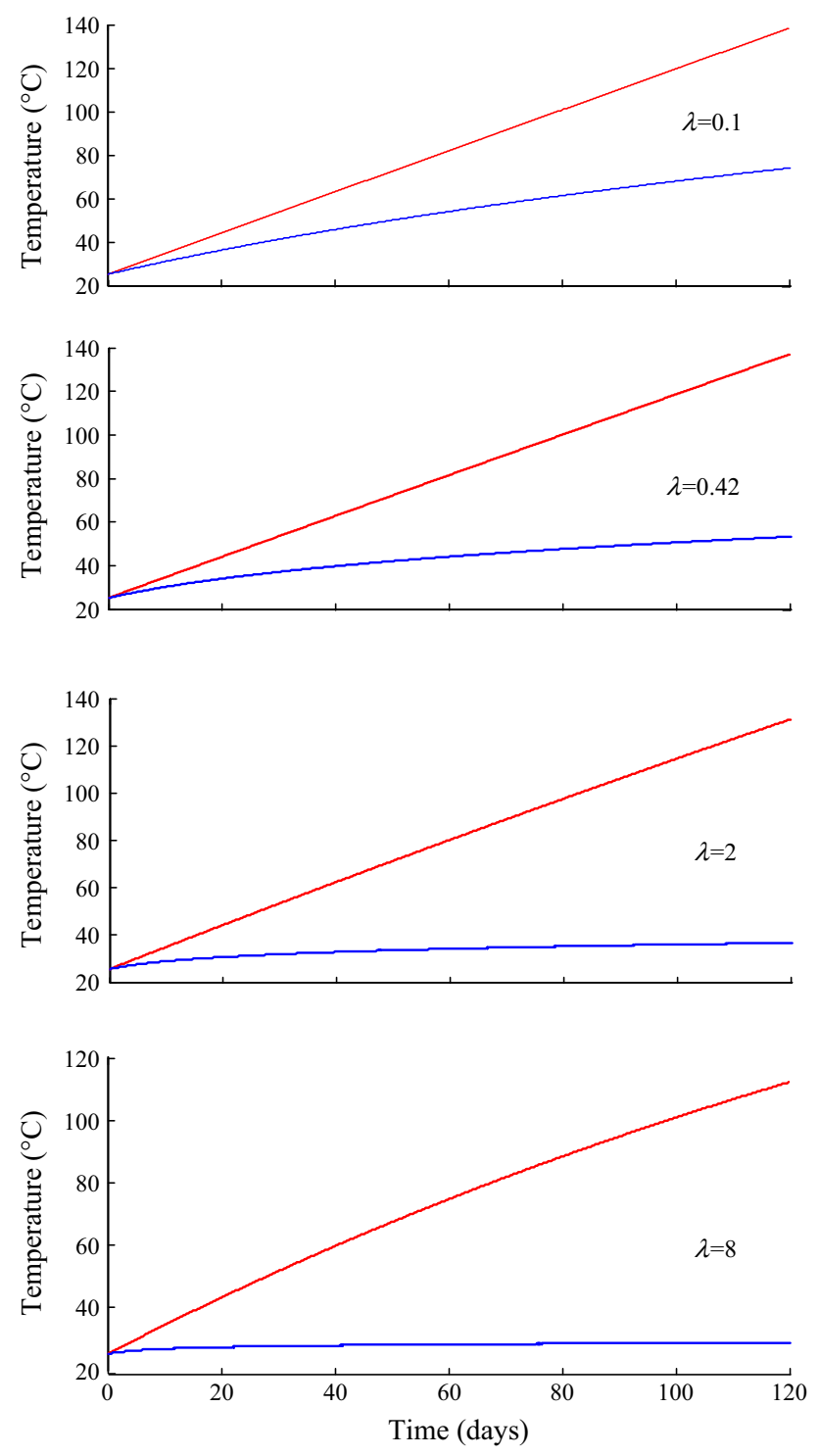

- Simulated results; — Experience formulas

Fig. 10 Temperature variation at the hottest point

\section{Conclusion}

This paper introduces the resistance network model for calculating the current field in the soil region around HVDC grounding electrodes. The method is highly applicable, and can be efficiently computed in practical HVDC projects.

Comparing the results of the resistance network method and the CDEGS, software the deviation between them is less than $5 \%$, which shows the accuracy of the resistance network method.

This paper also introduces the time-domain simulation of the temperature field based on FVM using the finite volume method, which is an alternative and more realistic way than previously published formulas to estimate the maximum soil temperature for the basic design in actual projects to the experience formulas. By taking into account the thermal conductivity of the soil the FVM avoids overestimating the soil temperature.

Open Access This article is distributed under the terms of the Creative Commons Attribution 4.0 International License (http://creativecommons.org/licenses/by/4.0/), which permits unrestricted use, distribution, and reproduction in any medium, provided you give appropriate credit to the original author(s) and the source, provide a link to the Creative Commons license, and indicate if changes were made.

\section{References}

[1] Takeda H, Ayakawa H, Tsumenaga M et al (1995) New protection method for HVDC lines including cables. IEEE Trans Power Deliver 10(4):2035-2039

[2] Yamaji K, Sato M, Kato K et al (1999) Cooperative control between large capacity HVDC system and thermal power plant. IEEE Trans Power Syst 14(2):629-634

[3] Giao TN, Sarma MP (1971) On the potential and field distribution around a ground electrode for HVDC transmission. IEEE Trans Power Appar Syst 90(6):2793-2801

[4] Meliopoulos APS, Christoforidis G (1989) Effects of DC ground electrode on converter transformers. IEEE Trans Power Deliver 9(4):58-59

[5] Tykeson K, Nyman A, Carlsson H (1996) Environmental and geographical aspects in HVDC electrode design. IEEE Trans Power Deliver 11(4):1948-1954

[6] Greiss H, Allen BL, Lagace PJ et al (1987) HVDC ground electrode heat dissipation. IEEE Trans Power Deliver 2(4):1008-1017

[7] Chen F, Zhang B, He JL (2008) Influence of coke bed on HVDC grounding electrode heat dissipation. IEEE Trans Magn 44(6):826-829

[8] Greiss H, Mukhedkar D, Houle JL et al (1988) HVDC ground electrode heat dissipation in an N-layer soil. IEEE Trans Power Deliver 3(4):1369-1374

[9] Chow YL, Yang JJ, Howard GE (1991) Complex images for electrostatic field computation in multilayered media. IEEE Trans Microw Theory 39(7):1120-1125

[10] Trlep M, Hamler A, Jesenik M et al (2003) The FEM-BEM analysis of complex grounding systems. IEEE Trans Magn 39(3-1):1155-1158

[11] Versteeg HK, Malalasekera W (1995) An introduction to computational fluid dynamics: The finite volume method. Wiley, New York

[12] Liu J, Dawalibi FP, Ma J, et al (2002) HVDC advanced analysis methods for grounding design and DC interference mitigation techniques. In: Proceedings of the 3 rd international symposium on electromagnetic compatibility (EMC'02), Beijing, 21-24 May 2002, pp 202-206

[13] Giao TN, Sarma MP (1972) Effect of a two-layer earth on the electric field near HVDC ground electrodes. IEEE Trans Power Appar Syst 91(6):2356-2365

[14] Dawalibi FP, Donoso F (1993) Integrated analysis software for grounding, EMF, and EMI. IEEE Comput Appl Power $6(2): 19-24$

[15] DL/T 5224-2005 technical rule for the design of HVDC earth return operation system(S). China Electric Power Press, Beijing (2005) (in Chinese) 
[16] Greiss H, Mukhedkar D, Lagace PJ (1989) Transient analysis of heat dissipation due to a HVDC ground electrode. IEEE Trans Power Deliver 4(2):916-920

Zheren ZHANG was born in Zhejiang, China, in October 1988. He received the B.S. degree in electrical engineering from Zhejiang University, Hangzhou, China, in 2011, where he is currently pursuing the $\mathrm{Ph} . \mathrm{D}$. degree in electrical engineering. His main field of interest is HVDC transmission.

Zheng XU was born in Zhejiang, China, in September 1962. He received the B.S., M.S., and Ph.D. degree in electrical engineering from Zhejiang University, Hangzhou, China, in 1983, 1986, and 1993, respectively. He has been with the Department of Electrical Engineering, Zhejiang University, since 1986 and has been a Professor there since 1998. His research area includes HVDC, FACTS, and grid integration of renewable energy.

Tao XU was born in Zhejiang, China, in February 1987. He received the B.S. and M.S. degree in electrical engineering from Zhejiang University, Hangzhou, China, in 2009, and 2012. He is now working for Zhejiang Electric Power Corporation Research Institute. His main field of interest is HVDC transmission. 